\title{
Prediction of a new body shape index and body adiposity estimator for development of type 2 diabetes mellitus: The Rural Chinese Cohort Study
}

\author{
Chengyi Han ${ }^{1,2,3}$, Yu Liu ${ }^{2}$, Xizhuo Sun ${ }^{2}$, Xinping Luo ${ }^{1}$, Lu Zhang ${ }^{1,4}$, Bingyuan Wang ${ }^{1,4}$, Yongcheng Ren ${ }^{1,4}$, \\ Junmei Zhou ${ }^{1}$, Yang Zhao ${ }^{1,4}$, Dongdong Zhang ${ }^{1,4}$, Xuejiao Liu ${ }^{1,4}$, Ming Zhang ${ }^{1}$ and Dongsheng $\mathrm{Hu}^{1,2 *}$ \\ ${ }^{1}$ Department of Preventive Medicine, Shenzhen University Health Sciences Center, 3688 Nanhai Avenue, Shenzhen, \\ Guangdong 518060, People's Republic of China \\ ${ }^{2}$ The Third Affiliated Hospital of Shenzhen University, 47 Youyi Road, Shenzhen, Guangdong 518001, People's \\ Republic of China \\ ${ }^{3}$ Department of Infection Control, The First Affiliated Hospital of Henan University of TCM, 19 Renmin Road, Zhengzhou, \\ Henan 450003, People's Republic of China \\ ${ }^{4}$ Department of Epidemiology and Health Statistics, College of Public Health, Zhengzhou University, 100 Kexue Avenue, \\ Zhengzhou, Henan 450001, People's Republic of China
}

(Submitted 11 March 2017 - Final revision received 9 September 2017 - Accepted 26 September 2017-First published online 16 November 2017)

\section{Abstract}

To compare the ability of a body shape index (ABSI) and body adiposity estimator (BAE) to BMI, waist circumference (WC) and waist:height ratio (WHtR) for predicting development of type 2 diabetes mellitus (T2DM) in rural adult Chinese. The prospective cohort study included 11687 eligible participants who were free of T2DM at baseline. The risk of new-onset T2DM for ABSI, BAE, BMI, WC and WHtR quintiles was estimated by Cox proportional-hazards regression at follow-up. We also compared the power of ABSI and BAE to BMI, WC and WHtR for predicting the development of T2DM. With increasing ABSI, BAE, BMI, WC and WHtR, T2DM incidence was substantially increased $\left(P_{\text {trend }}<0.0001\right)$. After adjustment for multi-covariates, risk of T2DM was increased from the second to fifth quintile as compared with first quintile for ABSI (1.27; $95 \%$ CI 0.95, 1.69; 1.35; $95 \%$ CI 1.00, 1.82; 1.75; $95 \%$ CI 1.33, 2.32 and 1.87; 95\% CI 1.40, 2.49; $\left.P_{\text {trend }}<0 \cdot 0001\right)$; BAE $\left(1 \cdot 82 ; 95 \%\right.$ CI $1.38,2 \cdot 41 ; 1.93 ; 95 \%$ CI $1.38,2.68 ; 2.73 ; 95 \%$ CI $1.94,3.84$ and $4.18 ; 95 \%$ CI $\left.2.98,5.87 ; P_{\text {trend }}<0.0001\right) ;$ BMI (1.42; $95 \%$ CI 1.03 , $1.97 ; 1.62 ; 95 \%$ CI $1.18,2 \cdot 23 ; 2.59 ; 95 \%$ CI $1.92,3.50$ and $3.90 ; 95 \%$ CI $\left.2.90,5 \cdot 26 ; P_{\text {trend }}<0.0001\right)$; WC (1.53; $95 \%$ CI $1.08,2 \cdot 17 ; 1 \cdot 66 ; 95 \%$ CI $1 \cdot 18,2 \cdot 33 ; 2 \cdot 72 ; 1 \cdot 97,3.76$ and $4.09 ; 95 \%$ CI $\left.2 \cdot 97,5.62 ; P_{\text {trend }}<0.0001\right)$; and WHtR (1.40; $95 \%$ CI 0.98, $1.99 ; 2 \cdot 06 ; 95 \%$ CI $1.47,2 \cdot 88 ; 2 \cdot 90 ; 95 \%$ CI $2 \cdot 10,4.01$ and $4.22 ; 95 \%$ CI 3.05, 5.85; $\left.P_{\text {trend }}<0.0001\right)$. ABSI, BAE, BMI, WC and WHR were effective and comparable in discriminating cases from non-cases of T2DM. Risk of T2DM was increased with elevated ABSI and BAE, but the predictive ability for T2DM did not differ than that of BMI, WC and WHtR in a rural Chinese population.

Key words: A body shape index: Body adiposity estimator: BMI: Waist circumstance: Waist:height ratio: Type 2 diabetes mellitus

In 2015, the International Diabetes Federation demonstrated that China has the highest number of adults with diabetes (109.6 million), and type 2 diabetes mellitus (T2DM) is the most common $^{(1)}$. However, many people with T2DM remain unaware of their condition for a long time because the symptoms are usually less marked than with type 1 diabetes mellitus and may take years to be recognised ${ }^{(2-4)}$. As a result, many people already have complications when they receive a diagnosis of $\mathrm{T}_{2} \mathrm{DM}^{(1)}$. About $80 \%$ of T2DM could be prevented by behaviour and lifestyle intervention $^{(5)}$. Therefore, identifying people at high risk of T2DM is indispensable for effective prevention strategies, which could help halt the inexorable increase in T2DM incidence.

Obesity is a confirmed risk factor of premature mortality ${ }^{(6,7)}$ and $\mathrm{T} 2 \mathrm{DM}^{(8)}$. To predict premature mortality better, Krakauer \& Krakauer $^{(9)}$, proposed a body shape index (ABSI) based on waist circumference (WC) adjusted for height and weight. ABSI could predict premature mortality better than traditional anthropometric measurements (BMI or WC) ${ }^{(10)}$. Recently, a prospective cohort study based on 687 urban Chinese found that ABSI was a weaker predictor of diabetes mellitus than traditional anthropometric measurements (BMI or WC), but the study participants were from a smaller urban population ${ }^{(11)}$. In addition, some studies concluded that excess adiposity but not excess body weight was the real culprit of $\mathrm{T}_{2} \mathrm{DM}^{(12,13)}$. To respond to the condition of unavailable body fat information in some studies, an estimation equation based on a large white population that accounted for BMI, sex and age to body fat has been developed $^{(14)}$. However, the predictive power of estimated body adiposity estimator (BAE) based on the equation for T2DM was not assessed in the Chinese population.

Abbreviations: ABSI, a body shape index; BAE, body adiposity estimator; T2DM, type 2 diabetes mellitus; WC, waist circumference; WHtR, waist:height ratio. 
The current study aimed to compare the power of ABSI and BAE with traditional anthropometric measurements (BMI, WC and waist:height ratio (WHtR)) for predicting new-onset T2DM in an adult Chinese population.

\section{Methods}

\section{Study design and participants}

A total of 19443 participants 18 to 74 years old were recruited from a rural Chinese population between July and August 2007 and July and August 2008; 16627 (85.5\%) participants completed the follow-up between July and August 2013 and July and October 2014. A questionnaire interview was used to collect data on demographic characteristics (sex and age), behavioural variables (smoking and drinking), physical activity and medical and family history by interviewing all participants face to face at baseline and follow-up. Among the 16627 participants who completed the follow-up, we excluded people who died ( $n$ 881), had T2DM ( $n$ 1260) and type 1 diabetes mellitus ( $n$ 11), lacked fasting plasma glucose (FPG) values ( $n$ 10) and anthropometric measurements ( $n$ 4), had BMI $<18.5 \mathrm{~kg} / \mathrm{m}^{2}$ ( $n$ 446) at baseline; and lacked FPG values ( $n$ 2328) at follow-up. Ultimately, 11687 participants were included in the analysis.

\section{Covariate measurement and definition}

A smoker was defined as having smoked 100 or more cigarettes during the lifetime ${ }^{(15)}$. An alcohol drinker was defined as having consumed alcohol twelve or more times in the past year ${ }^{(16)}$. Physical activity level was classified as high, moderate and low according to the International Physical Activity Questionnaire score ${ }^{(17)}$. Body weight and height were measured twice by use of a metric scale and a vertical height scale to the nearest $0.1 \mathrm{~kg}$ and $0.1 \mathrm{~cm}$, respectively, in participants wearing light indoor clothing without shoes. BMI was calculated as weight $(\mathrm{kg})$ divided by height $(\mathrm{m})$ squared. WC was analysed as the mean of two measurements to the nearest $0.1 \mathrm{~cm}$ by using a metric measurement tape at the $1-\mathrm{cm}$ surface level above the navel. WHtR was calculated as WC (m) divided by height (m). ABSI was defined as $\mathrm{WC} /\left((\mathrm{BMI})^{2 / 3} \times(\text { height })^{1 / 2}\right)^{(9)}$. BAE was defined as follows: $\mathrm{BAE}=-44.988+(0.503 \times$ age $)+(10.689 \times$ sex $)+$ $(3 \cdot 172 \times \mathrm{BMI})-\left(0.026 \times(\mathrm{BMI})^{2}\right)+(0 \cdot 181 \times \mathrm{BMI} \times \mathrm{sex})-(0 \cdot 02 \times$ $\mathrm{BMI} \times$ age $)-\left(0.005 \times(\mathrm{BMI})^{2} \times\right.$ sex $)+\left(0.00021 \times(\mathrm{BMI})^{2} \times\right.$ age $)$ where male $=0$ and female $=1$, and age is in years ${ }^{(14)}$. An electronic sphygmomanometer (HEM-770A; Fuzzy) was used to measure systolic blood pressure (SBP) and diastolic blood pressure (DBP) three times on the right arm of participants who were in a seated position after a 5-min rest and at 30-s intervals.

An overnight fasting venous blood sample was collected from each participant. Levels of lipids (total cholesterol (TC), TAG and HDL-cholesterol) and FPG were measured by using a HITACHI 7060 automatic biochemical analyzer. All interviews and examinations were performed by trained and dedicated study staff. This study was conducted according to the guidelines laid down in the Declaration of Helsinki and all procedures involving human subjects were approved by the Medical
Ethics Committee of Shenzhen University Health Sciences Center. Written informed consent was obtained from all participants.

\section{Definition of type 2 diabetes mellitus}

A diagnosis of T2DM was considered with a history of diabetes mellitus, currently using antidiabetic agents, or FPG level $\geq 7.0 \mathrm{mmol} / \mathrm{l}^{(18)}$. We excluded participants with type 1 diabetes mellitus, gestational diabetes and other diabetes types. The time of onset of T2DM was collected by dedicated survey staff who used a specially designed questionnaire with face-to-face interviews for participants. Person-years of follow-up were computed as the date of onset of T2DM or follow-up minus the date of baseline examination.

\section{Statistical analysis}

The Shapiro-Wilk test was used to evaluate normality of continuous variables. Data are presented as medians and interquartile ranges for continuous variables according to result of normality test and numbers and percentages for categorical variables. Wilcoxon's rank sum and $\chi^{2}$ tests were used to evaluate differences in continuous and categorical variables, respectively. The trend of T2DM incidence with increased ABSI, BAE, WC, BMI and WHtR was assessed by Cochran-MantelHaenzel test. Cox proportional-hazards regression analysis was used to estimate the risk of T2DM by anthropometric measurements (ABSI, WC, BMI and WHtR) and BAE adjusted for sex, age, smoking, alcohol consumption, physical activity, SBP and DBP, and TC, TAG and HDL-cholesterol levels. Simultaneously, the receiver operating characteristic (ROC) curve was used to identify cut-off values that balanced both sensitivity and specificity well for the anthropometric measurement in terms of the specific T2DM risk factor, with respective sensitivity and specificity values reported. All statistical analyses involved use of SAS 9.1 (SAS Institute), and a two-tailed $P<0.05$ was considered statistically significant. Software of Sample Size Calculations (Mark Woodward, The George Institute International Health; Lesley Francis, MIS Consultants Pty Ltd) was used estimated sample size. Statistical power was considered typically $80 \%$, two-sided significance level typically $0 \cdot 05$, T2DM incidence of unexposed group $0.5 \%$, relative risk $2 \cdot 0$ and exposed/ unexposed $0 \cdot 3$. These parameters were set to criteria from previous studies (incidence of unexposed group: $>0.5 \%$; relative risk: $>2)^{(19,20)}$ and overweight: $30 \cdot 1 \%$ ('Report on nutrition and non-communicated diseases in Chinese population $(2015)^{,(21)}$. Calculated result demonstrated the study need 12136 participants. Therefore, sample size was adequate in the study.

\section{Results}

The baseline characteristics of study participants with and without T2DM during follow-up are in Table 1. During a median of 6.01 years (interquartile range: 5.89-6.06) of follow-up, we recorded 749 new-onset T2DM. Data for age, BMI, WC, WHtR, 
Table 1. Baseline characteristics of study participants with and without type 2 diabetes mellitus (T2DM) during follow-up (Medians and interquartile ranges (IQR); numbers and percentages)

\begin{tabular}{|c|c|c|c|c|c|}
\hline \multirow[b]{2}{*}{ Variables } & \multicolumn{2}{|c|}{ With T2DM ( $n$ 749) } & \multicolumn{2}{|c|}{ Without T2DM (n 10 938) } & \multirow[b]{2}{*}{$P$} \\
\hline & Median & IQR & Median & IQR & \\
\hline Male & & & & & 0.8807 \\
\hline$n$ & \multicolumn{2}{|c|}{280} & \multicolumn{2}{|c|}{4119} & \\
\hline$\%$ & \multicolumn{2}{|c|}{37.38} & \multicolumn{2}{|c|}{37.66} & \\
\hline Age (years) & 53 & $44-60$ & 50 & $41-59$ & $<0.0001$ \\
\hline Tobacco consumption & & & & & 0.3420 \\
\hline$n$ & \multicolumn{2}{|c|}{187} & \multicolumn{2}{|c|}{2904} & \\
\hline$\%$ & \multirow{2}{*}{\multicolumn{2}{|c|}{24.97}} & \multicolumn{2}{|c|}{26.55} & \\
\hline Alcohol consumption & & & & & 0.7308 \\
\hline$n$ & \multicolumn{2}{|c|}{88} & \multicolumn{2}{|c|}{1240} & \\
\hline$\%$ & \multicolumn{2}{|c|}{11.75} & \multicolumn{2}{|c|}{11.34} & \\
\hline Physical activity & & & & & 0.1850 \\
\hline \multicolumn{6}{|l|}{ Low } \\
\hline$n$ & \multirow{2}{*}{\multicolumn{2}{|c|}{$\begin{array}{c}367 \\
49 \cdot 00\end{array}$}} & \multicolumn{2}{|c|}{5559} & \\
\hline$\%$ & & & & & \\
\hline \multicolumn{6}{|l|}{ Moderate } \\
\hline$n$ & \multirow{2}{*}{\multicolumn{2}{|c|}{154}} & \multicolumn{2}{|c|}{2386} & \\
\hline$\%$ & & & \multicolumn{2}{|c|}{$21 \cdot 81$} & \\
\hline \multicolumn{4}{|l|}{ High } & & \\
\hline$n$ & \multirow{2}{*}{\multicolumn{2}{|c|}{$\begin{array}{c}228 \\
30 \cdot 44\end{array}$}} & \multirow{2}{*}{\multicolumn{2}{|c|}{$\begin{array}{l}2993 \\
27.36\end{array}$}} & \\
\hline$\%$ & & & & & \\
\hline BMI $\left(\mathrm{kg} / \mathrm{m}^{2}\right)$ & $26 \cdot 39$ & $23 \cdot 88-28 \cdot 71$ & 24.08 & $21 \cdot 87-26 \cdot 49$ & $<0.0001$ \\
\hline WC $(\mathrm{cm})$ & 89.50 & $82 \cdot 10-96 \cdot 10$ & 81.75 & $75 \cdot 25-89.00$ & $<0.0001$ \\
\hline WHtR & 0.562 & $0.520-0.607$ & 0.517 & $0.474-0.562$ & $<0.0001$ \\
\hline ABSI $\left(\mathrm{m}^{11 / 6} \mathrm{~kg}^{-2 / 3}\right)$ & 0.080 & $0.077-0.083$ & 0.078 & $0.075-0.081$ & $<0.0001$ \\
\hline BAE & $35 \cdot 81$ & $28.09-40.41$ & $32 \cdot 15$ & $24.79-37.53$ & $<0.0001$ \\
\hline SBP $(\mathrm{mmol} / \mathrm{l})$ & 129.67 & $118 \cdot 33-142 \cdot 67$ & 121.67 & $111.00-135.33$ & $<0.0001$ \\
\hline $\mathrm{DBP}(\mathrm{mmol} / \mathrm{l})$ & 81.67 & $74.67-89.67$ & 77.33 & $70 \cdot 67-85.33$ & $<0.0001$ \\
\hline $\mathrm{TC}(\mathrm{mmol} / \mathrm{l})$ & 4.64 & $4.09-5 \cdot 23$ & $4 \cdot 36$ & $3.81-4.99$ & $<0.0001$ \\
\hline $\mathrm{TAG}(\mathrm{mmol} / \mathrm{l})$ & 1.85 & $1.24-2.69$ & 1.34 & $0.96-1.93$ & $<0.0001$ \\
\hline HDL-cholesterol $(\mathrm{mmol} / \mathrm{l})$ & 1.09 & $0.94-1.25$ & 1.14 & $0.99-1.32$ & $<0.0001$ \\
\hline $\mathrm{FPG}(\mathrm{mmol} / \mathrm{l})$ & 5.95 & $5 \cdot 44-6 \cdot 40$ & 5.29 & $4.96-5.63$ & $<0.0001$ \\
\hline
\end{tabular}

WC, waist circumference; WHtR, waist:height ratio; ABSI, a body shape index; BAE, body adiposity estimator; SBP, systolic blood pressure; DBP, diastolic blood pressure; TC, total cholesterol; FPG, fasting plasma glucose.

ABSI, BAE, SBP, DBP and TC, TAG and FPG levels were higher and HDL-cholesterol level was lower with T2DM than non-T2DM $(P<0.0001)$; the groups did not differ by sex, smoking, alcohol consumption or physical activity $(P>0.05)$.

Fig. 1 summarises the incidence and hazard ratios and $95 \%$ CI for T2DM by ABSI, BAE, BMI, WC and WHtR quintiles and continued value. T2DM incidence was increased with increasing level of ABSI, BAE, BMI, WC and WHtR $\left(P_{\text {trend }}<\right.$ 0.0001). As compared with the first quintile, with the second to fifth quintiles, the risk of T2DM increased for ABSI $(1.53 ; 95 \%$ CI $1 \cdot 16,2 \cdot 03 ; 1 \cdot 85 ; 95 \%$ CI $1 \cdot 38,2 \cdot 47 ; 2 \cdot 67 ; 95 \%$ CI $2 \cdot 05,3.48$ and 3.55 ; $95 \%$ CI 2.72, 4.64; $\left.P_{\text {trend }}<0.0001\right)$; BAE (1.97; $95 \%$ CI $1.50,2.59 ; 1.53 ; 95 \%$ CI $1.15,2.05 ; 2.36 ; 95 \%$ CI $1.81,3.09$ and $4.44 ; 95 \%$ CI $\left.3.46,5 \cdot 71 ; P_{\text {trend }}<0.0001\right)$; BMI (1.59; $95 \%$ CI 1.15 , $2 \cdot 21 ; 2.08 ; 95 \%$ CI $1.52,2 \cdot 84 ; 3.51 ; 95 \%$ CI 2.62, 4.69 and 5.95; $95 \%$ CI $\left.4.51,7 \cdot 86 ; P_{\text {trend }}<0 \cdot 0001\right) ;$ WC $(1 \cdot 78 ; 95 \%$ CI $1 \cdot 26,2 \cdot 53$; 2.19; $95 \%$ CI $1.57,3 \cdot 07$; 4.03; $95 \%$ CI $2 \cdot 95,5.51$ and $7 \cdot 00 ; 95 \%$ CI 5.19, 9.43; $\left.P_{\text {trend }}<0.0001\right)$; and WHtR (1.63; 95\% CI $1 \cdot 14$, $2 \cdot 31 ; 2 \cdot 67 ; 95 \%$ CI $1.93,3 \cdot 71 ; 4 \cdot 27 ; 95 \%$ CI $3 \cdot 14,5 \cdot 82$ and $7 \cdot 24$; $95 \%$ CI 5.38, 9.75; $\left.P_{\text {trend }}<0 \cdot 0001\right)$. After adjustment for sex, age, smoking, alcohol consumption, physical activity, SBP, DBP and TC, TAG and HDL-cholesterol levels, the association was consistent across the second to fifth quintiles for ABSI $(1 \cdot 27 ; 95 \% \mathrm{CI}$ $0.95,1.69 ; 1.35 ; 95 \%$ CI $1.00,1.82 ; 1.75 ; 95 \%$ CI $1.33,2.32$ and $1 \cdot 87 ; 95 \%$ CI $\left.1.40,2.49 ; P_{\text {trend }}<0.0001\right) ;$ BAE $(1 \cdot 82 ; 95 \%$ CI
$1 \cdot 38,2 \cdot 41 ; 1.93 ; 95 \%$ CI $1 \cdot 38,2 \cdot 68 ; 2 \cdot 73 ; 95 \%$ CI $1.94,3.84$ and $4 \cdot 18$; $95 \%$ CI $\left.2 \cdot 98,5 \cdot 87 ; P_{\text {trend }}<0 \cdot 0001\right)$; BMI (1.42; $95 \%$ CI 1.03 , $1.97 ; 1.62 ; 95 \%$ CI $1 \cdot 18,2 \cdot 23 ; 2.59 ; 95 \%$ CI $1.92,3.50$ and 3.90 ; $95 \%$ CI $\left.2 \cdot 90,5 \cdot 26 ; P_{\text {trend }}<0 \cdot 0001\right)$; WC $(1 \cdot 53 ; 95 \%$ CI $1 \cdot 08,2 \cdot 17$; $1.66 ; 95 \%$ CI $1.18,2 \cdot 33 ; 2 \cdot 72 ; 95 \%$ CI $1.97,3.76$ and $4.09 ; 95 \%$ CI $\left.2.97,5.62 ; P_{\text {trend }}<0.0001\right)$; and WHtR $(1.40 ; 95 \%$ CI 0.98 , $1.99 ; 2 \cdot 06 ; 95 \%$ CI $1.47,2 \cdot 88 ; 2.9095 \%$ CI $95 \%$ CI $2 \cdot 10,4.01$ and $4 \cdot 22 ; 95 \%$ CI 3.05, 5.85; $\left.P_{\text {trend }}<0 \cdot 0001\right)$. As continuous variables for ABSI, BAE, BMI, WC and WHtR, the risk of T2DM also increased in unadjusted and adjusted models $(P<0 \cdot 0001)$.

Baseline ABSI, BAE, BMI, WC and WHtR had moderate predictive ability for T2DM ( AUC (ROC)), sensitivity and specificity but high negative predictive value and low positive predictive value (Fig. 2 and Table 2). Specificity for ABSI and BAE for predicting T2DM was higher than traditional anthropometric measurements (BMI, WC and WHtR), but sensitivity, positive and negative predictive values, and AUC were lower (Fig. 2 and Table 2).

\section{Discussion}

We found risk of T2DM increased with elevated ABSI and BAE. However, for predicting development of T2DM, the ability of ABSI and BAE did not differ from traditional anthropometric measurements (BMI, WC and WHtR). 


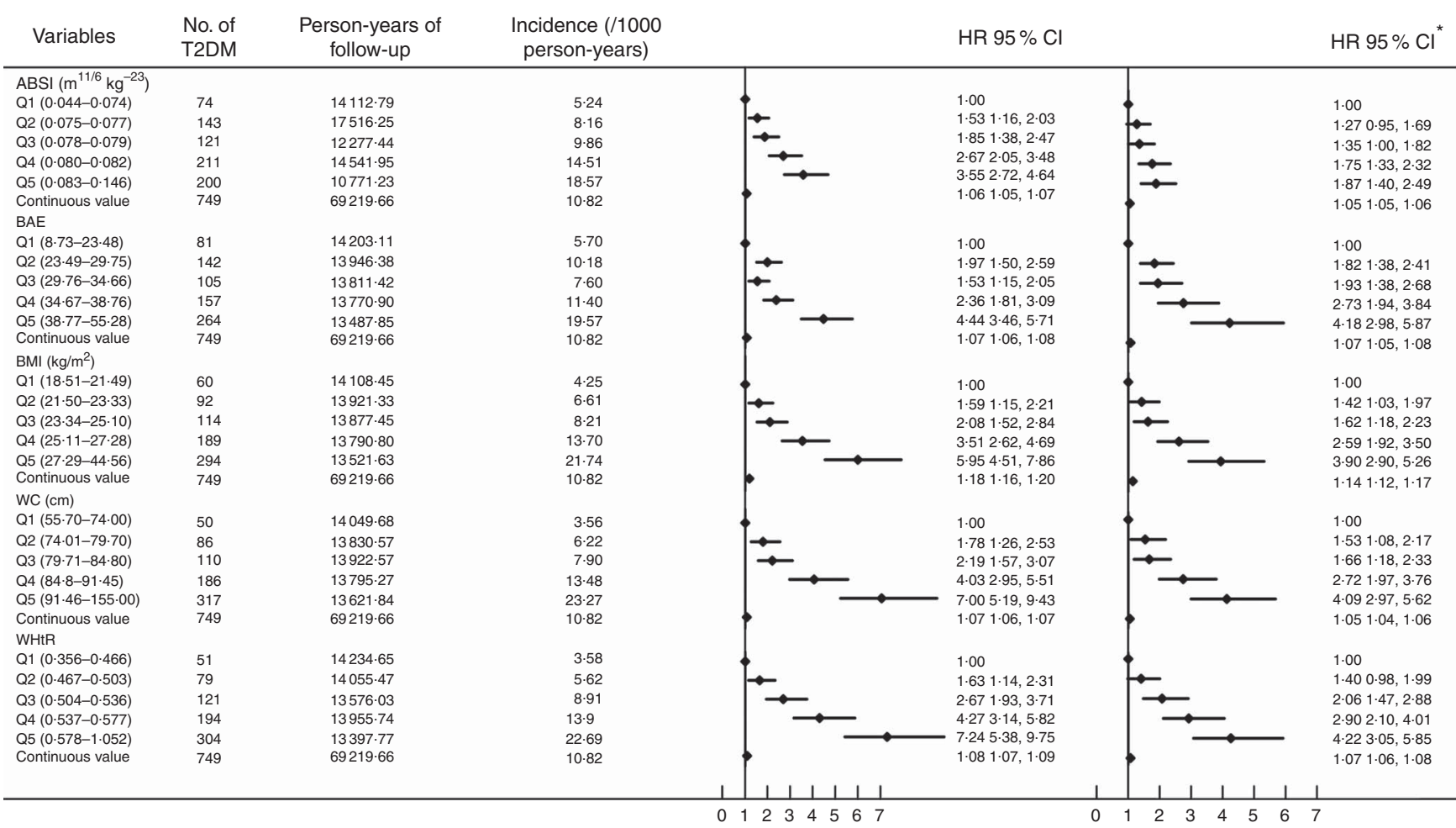

Fig. 1. Cox proportional-hazards analysis of predictors of type 2 diabetes mellitus (T2DM) incidence. ABSI, a body shape index; BAE, body adiposity estimator; HR, hazard ratio; WC, waist circumference; WHtR, waist:height ratio. * Adjusted for baseline sex, age, smoking, alcohol consumption, physical activity, systolic and diastolic blood pressure and total cholesterol, TAG and HDL-cholesterol levels.

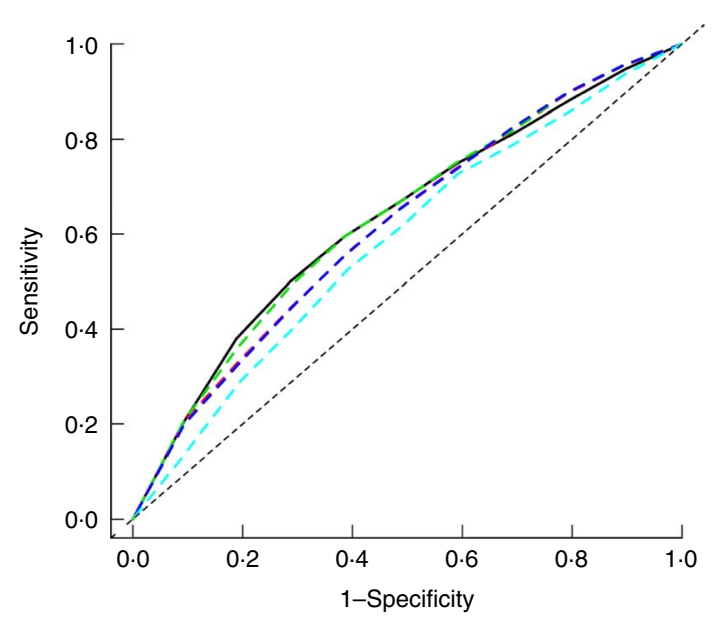

Fig. 2. Adjusted receiver operating characteristic $(\mathrm{ROC})$ curves for baseline factors for identifying type 2 diabetes mellitus (T2DM). _ Adjusted waist circumference (AUC 0.632); - - adjusted BMI (AUC 0.618); -- --, adjusted waist:height ratio (AUC 0.633); - - -, adjusted body adiposity estimator (AUC 0.618 ); — —, adjusted a body shape index (AUC 0.584). Adjusted for baseline sex, age, smoking, alcohol consumption, physical activity, systolic and diastolic blood pressure and total cholesterol, TAG and HDL-cholesterol levels.

Like traditional anthropometric measures (BMI, WC and WHtR $)^{(22-30)}$, elevated ABSI was associated with increased T2DM incidence. To our knowledge, only three cohort studies have evaluated the association of ABSI and diabetes mellitus ${ }^{(11,31,32)}$. One 15-year prospective cohort study of urban Chinese $(n$ 687) found ABSI independently associated with development of diabetes mellitus ${ }^{(11)}$. The 4-year retrospective cohort study of a Japanese population ( $n$ 37581) demonstrated elevated ABSI as an independent risk factor of diabetes mellitus but not a better predictor than $\mathrm{BMI}$ and $\mathrm{WC}^{(31)}$. Recently, the Atherosclerosis Risk in Communities (ARIC) study, with data for more than 11 years of follow-up, demonstrated that ABSI had a lower association and was an inferior discriminator of incident T2DM among all race-sex groups in a US population $^{(32)}$. A cross-sectional study based on 9555 Iranian adults suggested increased ABSI as an independent risk factor of abnormal glucose homoeostasis but not a better predictor than BMI, WHtR and $\mathrm{BAE}^{(33)}$. Previous studies indicated higher $\mathrm{WC}$ and $\mathrm{BMI}$ as an independent risk factor of $\mathrm{T}_{2} \mathrm{DM}^{(22,34)}$. ABSI is based on WC adjusted for height and weight. Therefore, high ABSI would be associated with increased T2DM incidence. A multicultural community-health assessment trial in Canada based on computed tomography demonstrated different amounts of abdominal adipose tissue among ethnic groups ${ }^{(35)}$. Development of ABSI, combining WC (mainly measurement of abdominal adipose tissue), height and weight, was based on the adult American ${ }^{(10)}$. Hence, ethnic differences might explain the weak predictive ability of ABSI for T2DM incidence.

We found general obesity (elevated BMI level) and central obesity (elevated WC) derived from excess adipose tissue as independent risk factors of T2DM incidence ${ }^{(35)}$, which is the best explanation for the relationship of increased BAE and T2DM incidence. Although the risk of T2DM did not differ with high BAE, WC or WHtR, BAE was not a better predictor of T2DM than WC and WHtR in our study. The predictive power of BAE for T2DM might be affected by some confounding 
Table 2. Power of baseline factors for predicting type 2 diabetes mellitus incidence (AUC (receiver operating characteristic) and $95 \%$ confidence intervals)

\begin{tabular}{|c|c|c|c|c|c|c|c|}
\hline Variables & Cut-off value & AUC & $95 \% \mathrm{Cl}$ & Sensitivity (\%) & Specificity (\%) & Positive predictive value (\%) & Negative predictive value (\%) \\
\hline ABSI $\left(m^{11 / 6} \mathrm{~kg}^{-2 / 3}\right)$ & 0.079 & 0.639 & $0.611,0.666$ & $57 \cdot 87$ & 64.66 & 8.88 & 95.65 \\
\hline $\mathrm{BAE}$ & $36 \cdot 05$ & 0.614 & $0.605,0.622$ & $49 \cdot 40$ & 68.09 & 9.57 & $95 \cdot 15$ \\
\hline BMI $\left(\mathrm{kg} / \mathrm{m}^{2}\right)$ & 24.98 & 0.689 & $0.661,0.715$ & 65.95 & 60.53 & $10 \cdot 24$ & $96 \cdot 28$ \\
\hline WC $(\mathrm{cm})$ & 83.35 & 0.704 & $0.677,0.730$ & $65 \cdot 60$ & 63.90 & 9.94 & $96 \cdot 58$ \\
\hline WHtR & 0.534 & 0.696 & $0.669,0.722$ & $68 \cdot 80$ & $60 \cdot 30$ & 10.54 & $96 \cdot 57$ \\
\hline
\end{tabular}

ABSI, a body shape index; BAE, body adiposity estimator; WC, waist circumstance; WHtR, waist:height ratio.

factors, whereas high BAE is an independent risk factor of T2DM. Because BAE is derived from a study of 6510 white participants $^{(14)}$, different ethnicity might be another explanation. Because of no additional study exploring BAE, WC and WHtR for predicting T2DM, further studies are still needed to confirm the possible relationship.

This analysis has several strengths, including its novel evaluation, relatively large sample size, community-based participants and face-to-face interviews. However, all participants were from rural areas, which might decrease the representativeness. We used only FPG to detect undiagnosed T2DM in this study, which may underestimate the incidence of $\mathrm{T} 2 \mathrm{DM}^{(36)}$. Many factors affect T2DM incidence. Because of lack of information on some factors, their potential effect on T2DM may not be fully eliminated, although we adjusted for multi-covariates by Cox regression analysis. Considering a $14.5 \%$ lost to follow-up, statistical analysis was performed on baseline demographic characteristics by response status. The results showed significant differences between responding and non-responding participants for age but not sex, which might suggest follow-up bias. Therefore, caution is warranted when interpreting and comparing the findings.

In conclusion, the risk of T2DM may be increased with elevated ABSI and BAE, but these factors are not better predictors of T2DM than BMI, WC or WHtR in rural Chinese population. Our findings may facilitate the development and promotion of preventive strategies aimed at obesity to reduce T2DM incidence.

\section{Acknowledgements}

The investigators are grateful to the dedicated participants and all research staff of the study.

This work was supported by the National Natural Science Foundation of China (grant nos 81373074, 81402752, 81673260); and the Science and Technology Development Foundation of Shenzhen (grant no. JCYJ20160307155707264).

C. H. and D. H.: design of the study. C. H., Y. L., X. S., X. L., L. Z., Y. R., B. W., J. Z. and Y. Z.: researched and analysed the data. C. H.: wrote the manuscript. D. Z., X. L., M. Z. and D. H.: contributed to the discussion and reviewed/edited the manuscript. All authors read and approved the final manuscript.

The authors declare that there are no conflicts of interest.

\section{References}

1. Lyons A (2016) Social support and the mental health of older gay men: findings from a national community-based survey. Res Aging 38, 234-253.
2. Li MZ, Su L, Liang BY, et al. (2013) Trends in prevalence, awareness, treatment, and control of diabetes mellitus in mainland China from 1979 to 2012. Int J Endocrinol 2013, 753150.

3. Rahman MS, Akter S, Abe SK, et al. (2015) Awareness, treatment, and control of diabetes in Bangladesh: a nationwide population-based study. PLOS ONE 10, e0118365.

4. Xu Y, Wang L, He J, et al. (2013) Prevalence and control of diabetes in Chinese adults. JAMA 310, 948-959.

5. Wang S, Marquez P, Langenbrunner J, et al. (2011) Toward a healthy and harmonious life in China: stemming the rising tide of non-communicable diseases. http://www.worldbank.org/ en/news/feature/2011/07/26/toward-health-harmonious-lifechina-stemming-rising-tide-of-non-communicable-diseases (accessed November 2016).

6. Global BMI Mortality Collaboration (2016) Body-mass index and all-cause mortality: individual-participant-data metaanalysis of 239 prospective studies in four continents. Lancet 388, 776-786.

7. Bellocco R, Jia C, Ye W, et al. (2010) Effects of physical activity, body mass index, waist-to-hip ratio and waist circumference on total mortality risk in the Swedish National March Cohort. Eur J Epidemiol 25, 777-788.

8. Haslam DW \& James WP. (2005) Obesity. Lancet 366, 1197-1209.

9. Krakauer NY \& Krakauer JC (2012) A new body shape index predicts mortality hazard independently of body mass index. PLOS ONE 7, e39504.

10. Krakauer NY \& Krakauer JC (2014) Dynamic association of mortality hazard with body shape. PLOS ONE 9, e88793.

11. He S \& Chen X (2013) Could the new body shape index predict the new onset of diabetes mellitus in the Chinese population? PLOS ONE 8, e50573.

12. Fruhbeck $G$ (2004) Screening and interventions for obesity in adults. Ann Intern Med 141, 245-246, author reply 246.

13. Thibault R \& Pichard C (2012) The evaluation of body composition: a useful tool for clinical practice. Ann Nutr Metab 60 , 6-16.

14. Gomez-Ambrosi J, Silva C, Catalan V, et al. (2012) Clinical usefulness of a new equation for estimating body fat. Diabetes Care 35, 383-388.

15. Tomar SL \& Asma S (2000) Smoking-attributable periodontitis in the United States: findings from NHANES III. National Health and Nutrition Examination Survey. J Periodontol 71, 743-751.

16. Gu D, He J, Duan X, et al. (2006) Body weight and mortality among men and women in China. JAMA 295, 776-783.

17. Craig CL, Marshall AL, Sjostrom M, et al. (2003) International physical activity questionnaire: 12-country reliability and validity. Med Sci Sports Exerc 35, 1381-1395.

18. IDF Clinical Guidelines Task Force (2005) Global guideline for type 2 diabetes. http://www.idf.org/global-guideline-type-2diabetes-2005 (accessed November 2016).

19. Zhang M, Wang B, Liu Y, et al. (2017) Cumulative increased risk of incident type 2 diabetes mellitus with increasing 
triglyceride glucose index in normal-weight people: The Rural Chinese Cohort Study. Cardiovasc Diabetol 16, 30

20. Ren Y, Liu Y, Sun X, et al. (2017) Hypertriglyceridemia-waist and risk of developing type 2 diabetes: The Rural Chinese Cohort Study. Sci Rep 7, 9072.

21. Gu J (2016) Report on nutrition and non-communicated diseases in Chinese population (2015) interpretation. Acta Nutrimenta Sinica 38, 525-529.

22. Su Y, Ma Y, Rao W, et al. (2016) Association between body mass index and diabetes in northeastern China: based on dose-response analyses using restricted cubic spline functions. Asia Pac J Public Health 28, 486-497.

23. Adegbija O, Hoy W \& Wang Z (2015) Predicting absolute risk of type 2 diabetes using age and waist circumference values in an aboriginal Australian community. PLOS ONE 10, e0123788.

24. Adegbija O, Hoy WE \& Wang Z (2015) Corresponding waist circumference and body mass index values based on 10-year absolute type 2 diabetes risk in an Australian aboriginal community. BMI Open Diabetes Res Care 3, e000127.

25. Chen F, Guo Z, Wu M, et al. (2015) Impact of dynamic changes of waist circumference and body mass index on type 2 diabetes mellitus risk. Zhonghua Yu Fang Yi Xue Za Zhi 49 , 1092-1097.

26. West RK, Ravona-Springer R, Heymann A, et al. (2016) Waist circumference is correlated with poorer cognition in elderly type 2 diabetes women. Alzheimers Dement 12, 925-929.

27. Yang XY, Zhang M, Luo XP, et al. (2016) Body mass index, waist circumference and waist-to-height ratio associated with the incidence of type 2 diabetes mellitus: a cohort study. Zhonghua Yu Fang Yi Xue Za Zbi 50, 328-333.

28. Kodama S, Horikawa C, Fujihara K, et al. (2012) Comparisons of the strength of associations with future type 2 diabetes risk among anthropometric obesity indicators, including waistto-height ratio: a meta-analysis. Am J Epidemiol 176, 959-969.
29. Lopatynski J, Mardarowicz G \& Szczesniak G (2003) A comparative evaluation of waist circumference, waist-to-hip ratio, waist-to-height ratio and body mass index as indicators of impaired glucose tolerance and as risk factors for type-2 diabetes mellitus. Ann Univ Mariae Curie Sklodowska Med 58, 413-419.

30. Xu Z, Qi X, Dahl AK, et al. (2013) Waist-to-height ratio is the best indicator for undiagnosed type 2 diabetes. Diabet Med 30, e201-e207.

31. Fujita M, Sato Y, Nagashima K, et al. (2015) Predictive power of a body shape index for development of diabetes, hypertension, and dyslipidemia in Japanese adults: a retrospective cohort study. PLOS ONE 10, e0128972.

32. Hardy DS, Stallings DT, Garvin JT, et al. (2017) Best anthropometric discriminators of incident type 2 diabetes among white and black adults: a longitudinal ARIC study. PLOS ONE 12, e0168282.

33. Haghighatdoost F, Sarrafzadegan N, Mohammadifard N, et al. (2014) Assessing body shape index as a risk predictor for cardiovascular diseases and metabolic syndrome among Iranian adults. Nutrition 30, 636-644.

34. Qiao Q \& Nyamdorj R (2010) Is the association of type ii diabetes with waist circumference or waist-to-hip ratio stronger than that with body mass index? Eur J Clin Nutr 64 , 30-34.

35. Lear SA, Humphries KH, Kohli S, et al. (2007) Visceral adipose tissue accumulation differs according to ethnic background: results of the Multicultural Community Health Assessment Trial (M-CHAT). Am J Clin Nutr 86, 353-359.

36. NCD Risk Factor Collaboration (NCD-RisC) (2015) Effects of diabetes definition on global surveillance of diabetes prevalence and diagnosis: a pooled analysis of 96 populationbased studies with 331,288 participants. Lancet Diabetes Endocrinol 3, 624-637. 